\title{
The Cumulative Effect of Stellar Encounters on Multi-Planet Systems in Star Clusters
}

\author{
Wei Hao ${ }^{1,2}$ and M. B. N. Kouwenhoven ${ }^{3}$ \\ ${ }^{1}$ Department of Astronomy, School of Physics, Peking University, Yi He Yuan Lu 5, Haidian \\ $\mathrm{Qu}$, Beijing 100871, P.R. China \\ email: elvis@MPA-Garching.MPG.DE \\ ${ }^{2}$ Max-Planck-Institut für Astrophysik, Karl-Schwarzschild-Str. 185741 Garching, Germany \\ ${ }^{3}$ Kavli Institute for Astronomy and Astrophysics (KIAA), Peking University, Yi He Yuan Lu \\ 5, Haidian Qu, Beijing 100871, P.R. China \\ email: kouwenhoven@pku.edu.cn
}

\begin{abstract}
Distant stellar encouters can substantially affect the dynamical evolution of existing stellar and planetary systems (e.g., Malmberg et al. 2007; Spurzem et al. 2009). Although planets with small orbital periods are not directly affected by encountering stars, the secular evolution of a perturbed system may result in the ejection of the innermost planets, or physical collisions between the innermost planets and the host star, hundreds of thousands of years after a weak encounter with a neighboring star occurs. Here we present the results of our study on the cumulative effect of distant stellar encounters on multi-planet systems in star clusters, and how these results depend on the properties of the star cluster in which a planetary system is born (for details we refer to Hao \& Kouwenhoven, in prep.). With our simulations we explain the scarcity of exoplanets in star clusters, not only for those in wide orbits (affected by stellar encounters), but also in close orbits (affected by the secular evolution of the system following an encounter).
\end{abstract}

Keywords. Planetary systems - open clusters and associations: general

\section{Introduction}

The majority of stars are formed in a clustered environment (Lada \& Lada 2003), and many of these stars are thought to form with a planetary system. Close and distant stellar encounters frequently occur (depending on the stellar density and lifetime of the star cluster). From observations of extra-solar planets we know that more than $10 \%$ of the nearby Solar-type stars have Jupiter-mass planets (Cumming et al. 2008). Recent observations with Kepler also indicate that a large number of the newly detected planets is part of a multi-planet system (Lissauer et al. 2011). Surpisingly, few planets are found in open clusters and globular clusters. Could this be the result of stellar encounters on existing systems?

\section{Method and initial conditions}

We study the environmental dependence on the evolution of multi-planet systems through scattering experiments. To maintain adequate energy conservation, $|\Delta E / E|<$ $10^{-8}$, we model the encounter phase using a modified version of the CHAIN package (Mikkola \& Aarseth 1993; Aarseth 2003) which is a fast and accurate algorithm for short-term energetic interactions in small $N$-body systems. For the time between two subsequent encounters we use the MERCURY symplectic integrator developed by Chambers 1999. We record changes in the orbital elements, properties of the ejected planets, and physical star-planet and planet-planet collisions. 

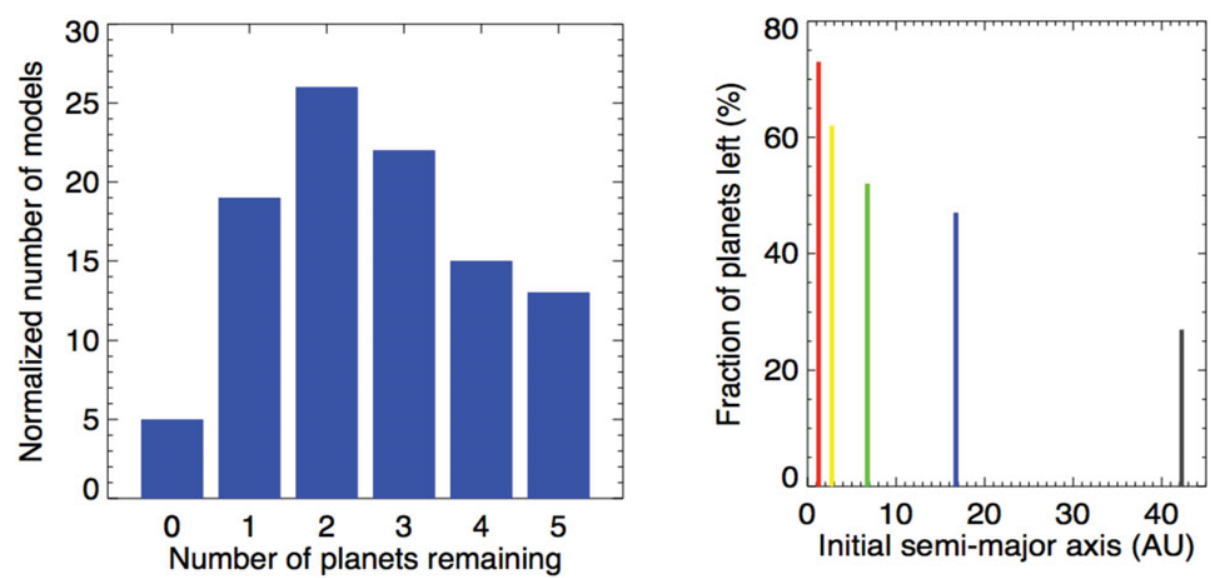

Figure 1. Survival properties of a simulated ensamble of multi-planet systems in a crowded stellar environment. Left: the number of surviving planets per system at the end of the simulations. Right: the survival fraction as a function of initial semi-major axis.

For our initial set-up of the planetary system we use the EMS (equal-mass equalseparation system) configuration (Zhou, Lin \& Sun 2007). Each system initially contains a $M_{\star}=1 M_{\odot}$ central star with five $M_{p}=1 M_{\text {Jup }}$ planets in co-planar and circular orbits. Following the theory of Kokubo \& Ida (2002), each consecutive pair of planets with semimajor axes $a_{i}$ and $a_{i+1}$ is initially separated by $10 R_{H}$, where $R_{H}=\left(2 M_{p} / 3 M_{\star}\right)^{1 / 3}\left(a_{i}+\right.$ $\left.a_{i+1}\right) / 2$ is their mutual Hill radius. The innermost planet is assigned a semi-major axis of $1 \mathrm{AU}$, somewhat within the snow-line, and the corresponding semi-major axes of the other planets are 2.6,6.5, 16.6, and 42.3 AU when adopting separations of $10 R_{H}$. The encountering star masses are drawn from the Chabrier (2003) initial mass function, their impact parameters $b$ from a distribution $d N / d b \propto b$, and their initial velocities from a Maxwellian velocity distribution appropriate for the star cluster that is modeled (see, e.g., Kouwenhoven et al. 2010). The interval between to successive encounters is drawn from a Poisson distribution. For demonstrative purposes we present here our results for an environment similar to that of the Orion Nebula Cluster, where each multi-planet system suffers 10-100 encounters within 1000 AU during the lifetime of the star cluster, which is typically several tens of millions of years.

Table 1. The dynamical fate of planets in crowded stellar environments. This table shows the fraction of escapers, collisions, and survivors for single-planet systems (columns 2-4) and multi-planet systems (columns 5-7).

\begin{tabular}{|c|ccc|ccc|}
\hline & \multicolumn{3}{|c|}{ Single-planet systems } & \multicolumn{3}{c|}{ Multi-planet systems } \\
\hline$a_{\text {initial }}$ (AU) & Escapers & Collisions & Survivors & Escapers & Collisions & Survivors \\
\hline 1.0 & $3 \%$ & $0 \%$ & $97 \%$ & $15 \%$ & $12 \%$ & $73 \%$ \\
2.6 & $7 \%$ & $0 \%$ & $93 \%$ & $31 \%$ & $7 \%$ & $62 \%$ \\
6.5 & $15 \%$ & $0 \%$ & $85 \%$ & $43 \%$ & $5 \%$ & $52 \%$ \\
16.6 & $46 \%$ & $0 \%$ & $54 \%$ & $51 \%$ & $2 \%$ & $47 \%$ \\
42.3 & $77 \%$ & $1 \%$ & $22 \%$ & $71 \%$ & $1 \%$ & $28 \%$ \\
\hline
\end{tabular}




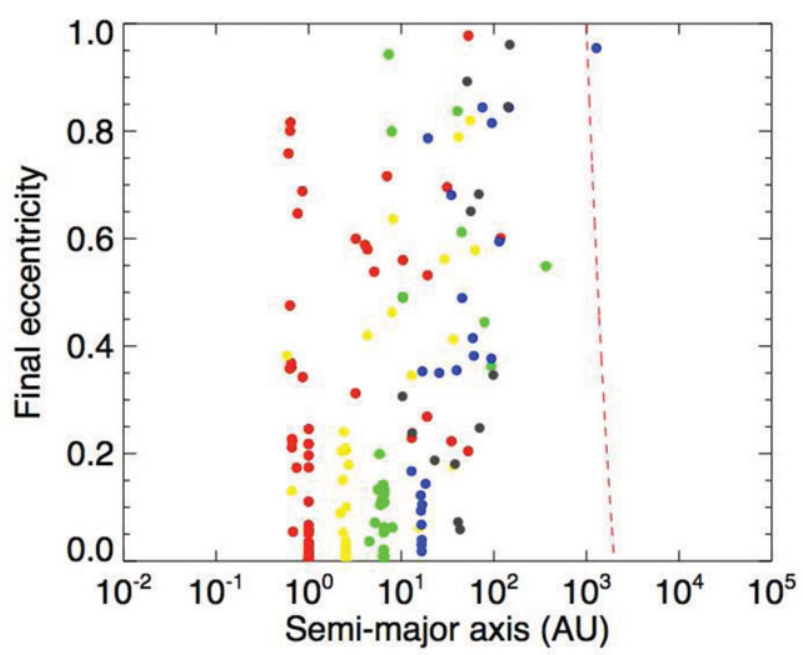

Figure 2. The semi-major axis $a$ and eccentricity $e$ of the planets at the end of the simulations. The dashed curve indicates the escaper removal criterion $a(1+e)>2000$ AU.

\section{Results and discussion}

We find that roughly half of the planets remain bound to their host star after suffering encounters with other stars in during the lifetime of the open cluster. The general properties of the outcome are presented in Figures 1 and 2. In this example we find that most of the inner planets $(a=1.0$ and $2.5 \mathrm{AU})$ survived but many of their orbital elements have changed. Most of the outer planets $(a=42.3 \mathrm{AU})$ are ejected from the system, while the few survivors have very wide and highly eccentric orbits. A substantial number of the surviving planets in wide orbits are highly inclined and occasionally have retrograde orbits. Table 1 shows the results of the simulations with a single planet versus simulations of multi-planet systems. Planetary systems with a single close-in planet are not easily perturbed in star clusters, as close encounters are rare. However, in the case of a multi-planet system, the secular evolution of a perturbed outer planetary system leads to perturbations, ejections, and physical star-planet and planet-planet collisions in the inner system. This suggests that the observed lack of close-in planets in star clusters may be the result of cumulative effect of distant encounters.

\section{References}

Aarseth, S. J. 2003, Gravitational N-Body Simulations, Cambridge University Press

Chabrier, G. 2003, PASP, 115, 763

Chambers, J. E. 1999, MNRAS, 304, 793

Cumming, A., Butler, R. P., Marcy, G. W., et al. 2008, PASP, 120, 531

Kokubo, E. \& Ida, S. 2002, ApJ, 581, 666

Kouwenhoven, M. B. N., Goodwin, S. P., Parker, R. J., et al. 2010, MNRAS, 404, 1835

Lada, C. J. \& Lada, E. A. 2003, ARAA, 41, 57

Lissauer, J. J., Ragozzine, D., Fabrycky, D. C., et al. 2011, ApJS, 197, 8

Malmberg, D., de Angeli, F., Davies, M. B., et al. 2007, MNRAS, 378, 1207

Mikkola, S. \& Aarseth, S. J. 1993, CeMDA, 57, 439

Spurzem, R., Giersz, M., Heggie, D. C., \& Lin, D. N. C. 2009, ApJ, 697, 458

Zhou, J.-L., Lin, D. N. C., \& Sun, Y.-S. 2007, ApJ, 666, 423 